\title{
Near-Field/Far-Field Transformation with Helicoidal Scanning from Irregularly Spaced Data
}

\author{
Francesco D’Agostino, Flaminio Ferrara, Claudio Gennarelli, Rocco Guerriero, \\ and Massimo Migliozzi
} Dipartimento di Ingegneria dell'Informazione ed Ingegneria Elettrica, University of Salerno, Via Ponte Don Melillo,
84084 Fisciano, Italy

Correspondence should be addressed to Claudio Gennarelli, gennar@diiie.unisa.it

Received 14 June 2010; Accepted 14 September 2010

Academic Editor: Krishnasamy Selvan

Copyright () 2010 Francesco D’Agostino et al. This is an open access article distributed under the Creative Commons Attribution License, which permits unrestricted use, distribution, and reproduction in any medium, provided the original work is properly cited.

\begin{abstract}
A fast and accurate technique for the compensation of the probe positioning errors in the near-field/far-field transformation with helicoidal scanning is proposed in this paper. It relies on a nonredundant sampling representation using a spherical modelling of the antenna under test and employs an iterative scheme to evaluate the near-field data at the points fixed by the helicoidal nonredundant representation from the acquired irregularly distributed ones. Once these helicoidal data have been recovered, those required by a classical cylindrical near-field/far-field transformation are efficiently determined by using an optimal sampling interpolation algorithm. Some numerical tests assessing the effectiveness of the proposed approach and its stability with respect to random errors affecting the near-field data are shown.
\end{abstract}

\section{Introduction}

As well-known, far-field (FF) range size limitations, transportation, and mounting problems can make impossible or impractical the measurement of antenna radiation patterns on a conventional FF range. In these cases, it is convenient to exploit near-field (NF) measurements and recover the FF patterns by using NF-FF transformation techniques [1-3]. In addition, the NF measurements may be performed in a controlled environment, as an anechoic chamber, thus overcoming those drawbacks that cannot be eliminated in FF outdoor measurements. In this framework, the reduction of the time needed for acquiring the NF data is assuming an ever growing relevance for the antenna measurement community, since this time is currently very much greater than that required to perform the corresponding NF-FF transformation. Such a reduction can be achieved by decreasing the number of the NF data to be collected and/or by making faster the acquisition of each NF value. A significant reduction of the number of required NF data has been obtained for all the conventional scannings [4-8] by applying the nonredundant sampling representations of electromagnetic (EM) fields and the optimal sampling interpolation (OSI) expansions [9], whereas, the use of the modulated scattering technique employing arrays of scattering probes has been proposed in [10] to realize a very fast electronic scanning. However, antenna testing NF facilities based on such a technique are not very flexible as those employing mechanical scans. These last can be made faster by exploiting innovative spiral scannings which use, as suggested by Yaccarino et al. in [11], continuous and synchronized movements of the positioning systems of the probe and antenna under test (AUT). In particular, accurate, stable, and efficient NF-FF transformations using the helicoidal scanning, the planar and spherical spiral scannings have been developed [1219]. They are based on the aforementioned nonredundant representations and reconstruct the NF data needed by the NF-FF transformation with the corresponding classical scanning by interpolating, via appropriate OSI formulas, the nonredundant samples acquired on the spiral. The required two-dimensional algorithm has been obtained (a) by assuming the AUT enclosed in a proper convex domain 
bounded by a surface $\Sigma$ with rotational symmetry; (b) by developing a nonredundant sampling representation of the voltage acquired by the probe on the spiral; (c) by choosing the spiral step equal to the spacing needed to interpolate the data along a meridian curve. In particular, the AUT has been assumed to be enclosed in the smallest sphere able to contain it in [12-15], whereas more effective AUT modellings, that allow a further reduction of required NF data when dealing with antennas having one or two predominant dimensions, have been adopted in [16-18] by properly applying the unified theory of spiral scannings for nonspherical antennas [19]. This last has been obtained by heuristically extending the rigorous approach for spherical antennas [15]. It is worth noting that these effective modellings allow one to consider measurement cylinders (planes) with a radius (distance) smaller than one half the antenna maximum size, thus reducing the error related to the truncation of the scanning zone.

Unfortunately, it may be impossible to get regularly distributed NF data due to an inaccurate control of the positioning systems, but the measurements points position can be accurately read by optical devices. In addition, the finite resolution of the positioning systems and their imprecise synchronization do not allow one to exactly locate the probe at the points fixed by the sampling representation. In the light of the above considerations, the development of an accurate and stable reconstruction procedure from irregularly spaced data becomes relevant. In this context, an approach based on the conjugate gradient iteration method and using the unequally spaced fast Fourier transform [20, 21] has been proposed in the planar [22] and spherical [23] classical scannings. However, such an approach is not tailored for scannings exploiting the nonredundant sampling representations of EM fields, wherein the "a priori" information on the AUT and proper sampling interpolations are employed to recover the NF data required by the corresponding standard NF-FF transformation technique. The interpolation from nonuniform samples has been well investigated in the onedimensional case of bandlimited functions defined over the real axis. Several sufficient conditions assuring the possibility of reconstructing a function from its nonuniform samples have been stated in [24]. The stability in a nonuniform sampling algorithm; that is, the requirement that small errors affecting the samples give rise to small errors in the reconstructed functions, has been exhaustively investigated in $[25,26]$, wherein it has been shown that a stable sampling cannot be accomplished at an "average" rate lower than the Nyquist one. Some closed-form expressions for the interpolation of bandlimited functions from nonuniform samples have been developed in [27]. However, they are valid only for particular sampling points arrangements, are cumbersome and not user friendly. Moreover, they become more and more unstable as the sampling points distribution deviates from the uniform one. The twodimensional nonuniform sampling has not attracted an equal consideration. In any case, it has been shown [28] that, again, a stable sampling cannot be performed at a rate lower than the Nyquist one. As it has been clearly stressed in [29], wherein a more exhaustive discussion on this topic can be found, a nonuniform sampling algorithm useful for practical applications must be computationally manageable, accurate, and stable. Accordingly, it is more convenient to recover the uniform samples from the irregularly distributed ones than to resort to a direct interpolation formula. In fact, once the uniform samples have been determined, the value at any point of the scanning surface can be recovered by an accurate and stable OSI expansion.

In this context, two different approaches have been proposed [29-31]. The former [29,30] is based on an iterative technique which has been found convergent only if there exists a one-to-one correspondence associating at each uniform sampling point the nearest nonuniform one. The latter [31] exploits the singular value decomposition (SVD) method [32] and has been applied when the twodimensional problem can be reduced to the research of the solution of two independent one-dimensional ones. This, f.i., occurs in a cylindrical near-field facility, wherein the nonuniformly distributed data can be realistically assumed to lie on not regularly spaced rings when the measurements are made by rings [31]. Such a hypothesis is no longer valid in the helicoidal scanning. Accordingly, the iterative technique will be here applied for reconstructing the uniformly spaced helicoidal samples from the acquired irregularly distributed data. Obviously, the SVD-based approach could be generalized to such an intrinsically two-dimensional problem, but the dimension of the involved matrix would become too large, thus requiring a huge computational effort.

In the following, the helicoidal scanning based on the spherical AUT modelling will be considered since it is more simple and general than those using modellings tailored for elongated antennas.

\section{Nonredundant Sampling Representation on a Cylinder}

Let us consider an AUT and a nondirective probe scanning a helix with constant angular step lying on a cylinder of radius $d$ (Figure 1) and adopt the spherical coordinate system $(r, \vartheta, \varphi)$ to denote an observation point $P$ in the NF region. Since, as shown in [33], the voltage measured by such a kind of probe has the same effective spatial bandwidth of the AUT field, the nonredundant sampling representations of EM fields [9] can be applied to it. Accordingly, by assuming the AUT as enclosed in the smallest sphere of radius $a$ able to contain it and describing the helix by a proper analytical parameterization $\underline{r}=\underline{r}(\xi)$, it is possible to consider the "reduced voltage"

$$
\tilde{V}(\xi)=V(\xi) \mathrm{e}^{\mathrm{j} \gamma(\xi)},
$$

where $\gamma(\xi)$ is an optimal phase function to be determined. The error, occurring when $\tilde{V}$ is approximated by a spatially bandlimited function, becomes negligible as the bandwidth exceeds a critical value $W_{\xi}$ [9], so that it can be effectively controlled by choosing a bandwidth equal to $\chi^{\prime} W_{\xi}$, where $\chi^{\prime}$ is an enlargement factor, slightly greater than unity for electrically large antennas. 


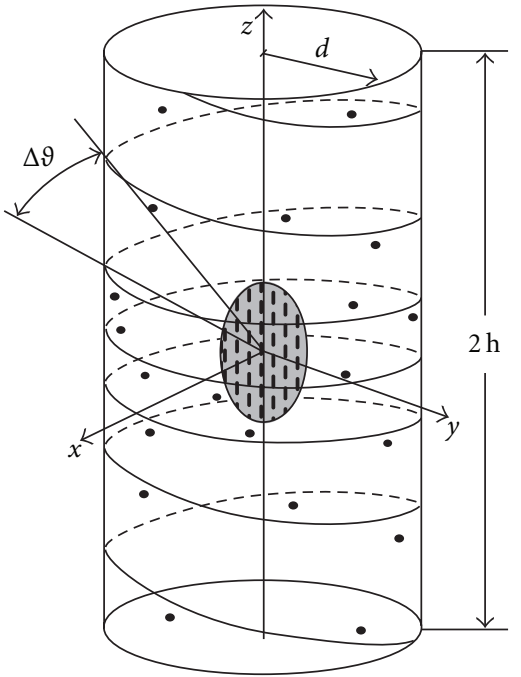

Figure 1: Geometry of the problem.

The parametric equation of the helix, when imposing its passage through a fixed point $P_{0}$ of the generatrix at $\varphi=0$, are

$$
\begin{aligned}
& x=d \cos \left(\phi-\phi_{s}\right), \\
& y=d \sin \left(\phi-\phi_{s}\right), \\
& z=d \cot \theta,
\end{aligned}
$$

where $\phi$ is the angular parameter describing the helix, $\phi_{s}$ is the value of $\phi$ at $P_{0}$, and $\theta=k \phi$. Such a helix can be constructed as intersection of the cylinder with the line from the origin to a point moving on a spiral which wraps the sphere enclosing the AUT. In order to allow the twodimensional interpolation, the helix step $\Delta \theta$ (Figure 1) must be equal to the spacing required for the voltage interpolation along a generatrix [6]. Therefore, the parameter $k$ is such that the angular step, determined by the consecutive intersections of the helix with a generatrix, is $\Delta \theta=2 \pi /\left(2 N^{\prime \prime}+1\right)$, with $N^{\prime \prime}=\operatorname{Int}\left(\chi N^{\prime}\right)+1$ and $N^{\prime}=\operatorname{Int}\left(\chi^{\prime} \beta a\right)+1$. Accordingly, being $\Delta \theta=2 \pi k$, it results that $k=1 /\left(2 N^{\prime \prime}+1\right)$. The function $\operatorname{Int}(x)$ denotes the integer part of $x, \beta$ is the wavenumber, and $\chi>1$ is an oversampling factor needed for controlling the truncation error [9].

A nonredundant sampling representation of the voltage on the helix can be obtained by using the following expressions for the phase function and parameterization [15]

$$
\begin{aligned}
& \gamma=\beta \int_{0}^{r} \sqrt{1-\frac{a^{2}}{r^{\prime 2}}} \mathrm{~d} r^{\prime}=\beta \sqrt{r^{2}-a^{2}}-\beta a \cos ^{-1}\left(\frac{a}{r}\right), \\
& \xi=\frac{\beta a}{W_{\xi}} \int_{0}^{\phi} \sqrt{k^{2}+\sin ^{2} k \phi^{\prime}} \mathrm{d} \phi^{\prime} .
\end{aligned}
$$

As can be seen, the optimal parameter $\xi$ is proportional to the curvilinear abscissa along the spiral wrapping the sphere modelling the AUT. Since such a spiral is a closed curve, it is convenient to choose the bandwidth $W_{\xi}$ such that $\xi$ covers a $2 \pi$ range when the whole curve on the AUT sphere is described. Therefore,

$$
W_{\xi}=\frac{\beta a}{\pi} \int_{0}^{\left(2 N^{\prime \prime}+1\right) \pi} \sqrt{k^{2}+\sin ^{2} k \phi^{\prime}} \mathrm{d} \phi^{\prime} .
$$

In the light of these results, the reduced voltage at any point of the helix can be reconstructed via the OSI expansion:

$$
\tilde{V}(\xi)=\sum_{m=m_{0}-p+1}^{m_{0}+p} \tilde{V}\left(\xi_{m}\right) \Omega_{M}\left(\xi-\xi_{m}\right) D_{M^{\prime \prime}}\left(\xi-\xi_{m}\right),
$$

where $m_{0}=\operatorname{Int}\left[\left(\xi-\xi_{s}\right) / \Delta \xi\right]$ is the index of the sample nearest (on the left) to the output point, $2 p$ is the number of retained samples $\tilde{V}\left(\xi_{m}\right)$, and

$$
\xi_{m}=\xi\left(\phi_{s}\right)+m \Delta \xi=\xi_{s}+\frac{2 \pi m}{\left(2 M^{\prime \prime}+1\right)}
$$

with $M^{\prime \prime}=\operatorname{Int}\left(\chi M^{\prime}\right)+1, M^{\prime}=\operatorname{Int}\left(\chi^{\prime} W_{\xi}\right)+1$, and $M=$ $M^{\prime \prime}-M^{\prime}$. Moreover,

$$
\begin{gathered}
D_{M^{\prime \prime}}(\xi)=\frac{\sin \left(\left(2 M^{\prime \prime}+1\right) \xi / 2\right)}{\left(2 M^{\prime \prime}+1\right) \sin (\xi / 2)}, \\
\Omega_{M}(\xi)=\frac{T_{M}\left[2(\cos (\xi / 2) / \cos (\bar{\xi} / 2))^{2}-1\right]}{T_{M}\left[2 / \cos ^{2}(\bar{\xi} / 2)-1\right]}
\end{gathered}
$$

are the Dirichlet and Tschebyscheff Sampling functions, respectively, $T_{M}(\cdot)$ being the Tschebyscheff polynomial of degree $M$ and $\bar{\xi}=p \Delta \xi$.

The OSI expansion (5) can be used to evaluate the "intermediate samples"; that is, the reduced voltage values at the intersection points between the helix and the generatrix passing through $P$. Once these samples have been evaluated, the reduced voltage at $P$ can be recovered via the following OSI formula:

$$
\tilde{\mathrm{V}}(\vartheta, \varphi)=\sum_{n=n_{0}-q+1}^{n_{0}+q} \tilde{\mathrm{V}}\left(\vartheta_{n}\right) D_{N^{\prime \prime}}\left(\vartheta-\vartheta_{n}\right) \Omega_{\mathrm{N}}\left(\vartheta-\vartheta_{n}\right),
$$

where $n_{0}=\operatorname{Int}\left[\left(\vartheta-\vartheta_{0}\right) / \Delta \vartheta\right], N=N^{\prime \prime}-N^{\prime}, \tilde{V}\left(\vartheta_{n}\right)$ are the intermediate samples

$$
\vartheta_{n}=\vartheta_{n}(\varphi)=\vartheta\left(\phi_{s}\right)+k \varphi+n \Delta \vartheta=\vartheta_{0}+n \Delta \vartheta
$$

and the other symbols have the same meaning as in (5). It is so possible to recover the NF data required to perform the standard NF-FF transformation with cylindrical scanning [34], whose key steps are reported in the next section for reader's convenience.

\section{Probe Compensated NF-FF Transformation}

As rigorously demonstrated in [34], the modal coefficients $a_{v}$ and $b_{v}$ of the cylindrical wave expansion of the field radiated by the AUT are related to (a) the two-dimensional Fourier transforms $I_{v}$ and $I_{v}^{\prime}$ of the output voltage of the probe for two independent sets of measurements (the probe 
is rotated $90^{\circ}$ about its longitudinal axis in the second set); (b) the coefficients $c_{m}, d_{m}$ and $c_{m}^{\prime}, d_{m}^{\prime}$ of the cylindrical wave expansion of the field radiated by the probe and the rotated probe, respectively, when used as transmitting antennas. In particular,

$$
\begin{aligned}
& a_{\nu}(\eta)= \frac{\beta^{2}}{\Lambda^{2} \Delta_{\nu}(\eta)} \\
& \times\left[I_{\nu}(\eta) \sum_{m=-\infty}^{\infty} d_{m}^{\prime}(-\eta) H_{\nu+m}^{(2)}(\Lambda d)\right. \\
&\left.-I_{\nu}^{\prime}(\eta) \sum_{m=-\infty}^{\infty} d_{m}(-\eta) H_{\nu+m}^{(2)}(\Lambda d)\right] \\
& b_{\nu}(\eta)= \frac{\beta^{2}}{\Lambda^{2} \Delta_{\nu}(\eta)} \\
& \times\left[I_{\nu}^{\prime}(\eta) \sum_{m=-\infty}^{\infty} c_{m}(-\eta) H_{\nu+m}^{(2)}(\Lambda d)\right. \\
&\left.-I_{\nu}(\eta) \sum_{m=-\infty}^{\infty} c_{m}^{\prime}(-\eta) H_{\nu+m}^{(2)}(\Lambda d)\right] \\
& I_{\nu}(\eta)= \int_{-\infty}^{\infty} \int_{-\pi}^{\pi} V(\varphi, z) \mathrm{e}^{-\mathrm{j} \nu \varphi} \mathrm{e}^{\mathrm{j} \eta z} \mathrm{~d} \varphi \mathrm{d} z, \\
& I_{\nu}^{\prime}(\eta)= \sum_{-\infty}^{\infty} \int_{-\pi}^{\pi} V^{\prime}(\varphi, z) \mathrm{e}^{-\mathrm{j} \nu \varphi} \mathrm{e}^{\mathrm{j} \eta z} \mathrm{~d} \varphi \mathrm{d} z \\
& I_{\nu}^{\prime}(-\eta) H_{\nu+m}^{(2)}(\Lambda d) \sum_{m=-\infty}^{\infty} d_{m}(-\eta) H_{\nu+m}^{(2)}(\Lambda d) \\
& \sum_{m=-\infty}^{\infty} c_{m}(-\eta) H_{\nu+m}^{(2)}(\Lambda d) \sum_{m=-\infty}^{\infty} d_{m}^{\prime}(-\eta) H_{\nu+m}^{(2)}(\Lambda d)
\end{aligned}
$$

where $\Lambda=\sqrt{\beta^{2}-\eta^{2}}, H_{\nu}^{(2)}(\cdot)$ is the Hankel function of second kind and order $v$, and $V, V^{\prime}$ represent the output voltage of the probe and the rotated probe at the point of cylindrical coordinates $(d, \varphi, z)$.

Once the modal coefficients have been determined, the FF components of the electric field in the spherical coordinate system $(R, \Theta, \Phi)$ can be evaluated by

$$
\begin{aligned}
& E_{\Theta}=-j 2 \beta \frac{\mathrm{e}^{-j \beta R}}{R} \sin \Theta \sum_{\nu=-\infty}^{\infty} j^{\nu} b_{\nu}(\beta \cos \Theta) e^{j \nu \Phi}, \\
& E_{\Phi}=-2 \beta \frac{e^{-j \beta R}}{R} \sin \Theta \sum_{\nu=-\infty}^{\infty} j^{\nu} a_{\nu}(\beta \cos \Theta) e^{j \nu \Phi} .
\end{aligned}
$$

\section{Uniform Samples Reconstruction}

Let us now suppose that the samples are irregularly distributed (Figure 1) and denote with $\left(\bar{\vartheta}_{i}, \bar{\varphi}_{i}\right)$ the nonuniform sampling point corresponding to the nearest uniform one $\xi_{i}$ lying on the helix. By expressing the reduced voltage at each nonuniform sampling point as a function of the unknown

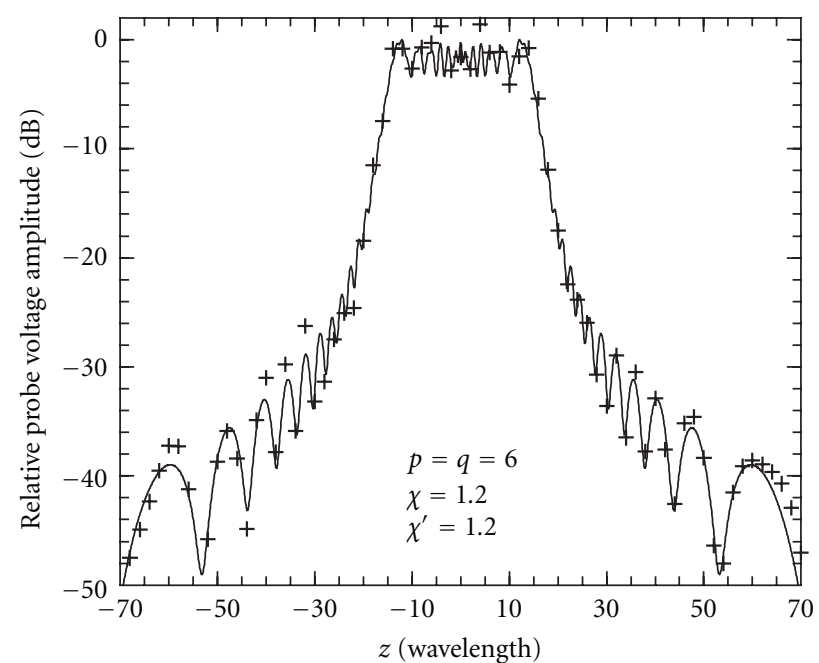

Figure 2: Amplitude of the probe voltage $V$ on the generatrix at $\varphi=90^{\circ}$. Solid line: exact. Crosses: recovered from irregularly spaced NF data at the iteration 0 .

values at the nearest uniform ones via the following twodimensional OSI formula (obtained by properly merging expansions (5) and (9)), it results in

$$
\begin{array}{r}
\tilde{\mathrm{V}}\left(\bar{\vartheta}_{i}, \bar{\varphi}_{i}\right) \\
=\sum_{n=n_{0}-q+1}^{n_{0}+q}\left[D_{N^{\prime \prime}}\left(\bar{\vartheta}_{i}-\vartheta_{n}\right) \Omega_{N}\left(\bar{\vartheta}_{i}-\vartheta_{n}\right)\right. \\
\left.\cdot \sum_{m=m_{0}-p+1}^{m_{0}+p} \tilde{V}\left(\xi_{m}\right) \Omega_{M}\left(\xi\left(\vartheta_{n}\right)-\xi_{m}\right) D_{M^{\prime \prime}}\left(\xi\left(\vartheta_{n}\right)-\xi_{m}\right)\right] \\
i=1,2, \ldots, Q,
\end{array}
$$

where $Q$ is the number of samples. The system (13) can be recast in the matrix form

$$
\underline{\underline{A}} \underline{x}=\underline{b}
$$

where $\underline{\underline{A}}$ is a $Q \times Q$ sparse banded matrix whose elements are given by

$$
\begin{aligned}
A_{i m}= & D_{N^{\prime \prime}}\left(\bar{\vartheta}_{i}-\vartheta_{n}\right) \Omega_{N}\left(\bar{\vartheta}_{i}-\vartheta_{n}\right) \\
& \times D_{M^{\prime \prime}}\left(\xi\left(\vartheta_{n}\right)-\xi_{m}\right) \Omega_{M}\left(\xi\left(\vartheta_{n}\right)-\xi_{m}\right),
\end{aligned}
$$

where $\underline{b}$ is the vector of the collected nonuniform data, and $\underline{x}$ is the vector of the unknown uniform samples.

By splitting $\underline{\underline{A}}$ in its diagonal and nondiagonal parts, $\underline{\underline{A}}_{D}$ and $\triangleq$, respectively, multiplying both members of (14) by $\underline{\underline{A}}_{D}^{-1}$ and rearranging the terms, it results in

$$
\underline{x}=\underline{\underline{A}}_{D}^{-1} \underline{b}-\underline{\underline{A}}_{D}^{-1} \triangleq \underline{\underline{x}} .
$$

The following iterative scheme is so obtained:

$$
\underline{x}^{(\nu)}=\underline{\underline{A}}_{D}^{-1} \underline{b}-\underline{\underline{A}}_{D}^{-1} \triangleq \underline{x}^{(\nu-1)}=\underline{x}^{(0)}-\underline{\underline{A}}_{D}^{-1} \triangleq \underline{x}^{(\nu-1)},
$$


where $\underline{x}^{(\nu)}$ is the vector of the uniform samples estimated at the $\nu$ th step. Necessary conditions for the convergence of such a scheme $[29,30]$ are that $A_{i i} \neq 0$, for all $i$, and $\left|A_{i i}\right| \geq$ $\left|A_{\text {im }}\right|$, for all $m \neq i$. These conditions are surely verified in the assumed hypothesis of biunique correspondence between each uniform sampling point and the "nearest" nonuniform one.

By straightforward evaluations from (17), it results

$$
\begin{aligned}
& \tilde{V}^{(v)}\left(\xi_{i}\right) \frac{1}{A_{i i}}\left\{\tilde{V}\left(\bar{\vartheta}_{i}, \bar{\phi}_{i}\right)\right. \\
&-\sum_{n=n_{0}-q+1} {\left[\Omega_{N}\left(\bar{\vartheta}_{i}-\vartheta_{n}\right) D_{N^{\prime \prime}}\left(\bar{\vartheta}_{i}-\vartheta_{n}\right)\right.} \\
& \cdot \sum_{\substack{m=m_{0}-p+1 \\
m \neq i}}^{n_{0}+p} \tilde{V}^{(\nu-1)}\left(\xi_{m}\right) \Omega_{M}\left(\xi\left(\vartheta_{n}\right)-\xi_{m}\right) \\
&\left.\left.\times D_{M^{\prime \prime}}\left(\xi\left(\vartheta_{n}\right)-\xi_{m}\right)\right]\right\} \quad i=1,2, \ldots, Q,
\end{aligned}
$$

where

$$
\begin{aligned}
A_{i i}= & D_{N^{\prime \prime}}\left(\bar{\vartheta}_{i}-\vartheta_{n_{i}}\right) \Omega_{N}\left(\bar{\vartheta}_{i}-\vartheta_{n_{i}}\right) \\
& \times D_{M^{\prime \prime}}\left(\xi\left(\vartheta_{n}\right)-\xi_{m}\right) \Omega_{M}\left(\xi\left(\vartheta_{n}\right)-\xi_{m}\right)
\end{aligned}
$$

$n_{i}=\operatorname{Nint}\left[\left(\bar{\vartheta}_{i}-\vartheta_{0}\right) / \Delta 9\right]$ being the index of the intermediate sampling point nearest to the uniform one $\xi_{i}$.

\section{Numerical Results}

The effectiveness and robustness of the proposed algorithm for compensating the probe positioning errors in the NF-FF transformation with helicoidal scanning have been assessed by many numerical tests. The reported simulations refer to a uniform circular array lying on the plane $y=0$ (see Figure 1), symmetric with respect to the plane $z=0$ and having radius $a=16 \lambda, \lambda$ being the wavelength. Its elements are elementary Huygens sources polarized along the $z$ axis and are radially and azimuthally spaced of $\lambda / 2$. An open-ended WR-90 rectangular waveguide, operating at the frequency of $10 \mathrm{GHz}$, is chosen as probe. The considered helix wraps a cylinder with radius $d=20 \lambda$ and height $2 h=140 \lambda$. The irregularly distributed samples have been generated by imposing that the distances in $\xi$ and $\vartheta$ between the position of each nonuniform sample and the associate uniform one are random variables uniformly distributed in $(-0.3 \Delta \xi, 0.3 \Delta \xi)$ and $(-0.3 \Delta \vartheta, 0.3 \Delta \vartheta)$. Note that this represents a pessimistic occurrence in a real scanning procedure.

Figures 2 and 3 show a representative reconstruction example of the output voltage $V$ (the most significant one) on the generatrix at $\varphi=90^{\circ}$, obtained by 0 and 8 iterations, respectively. As it can be seen, only 8 iterations are

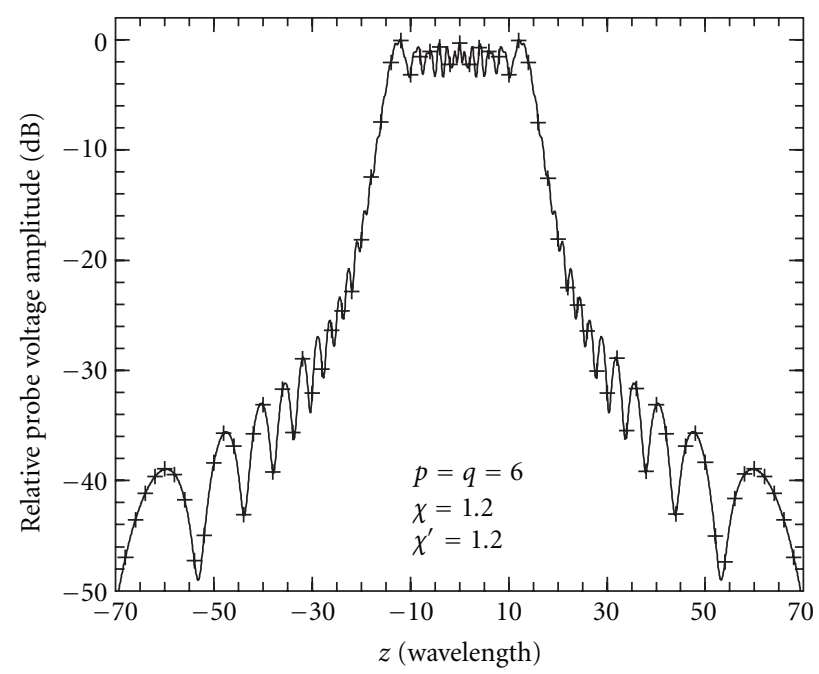

FIGURE 3: Amplitude of the probe voltage $V$ on the generatrix at $\varphi=90^{\circ}$. Solid line: exact. Crosses: recovered from irregularly spaced NF data at the iteration 8 .

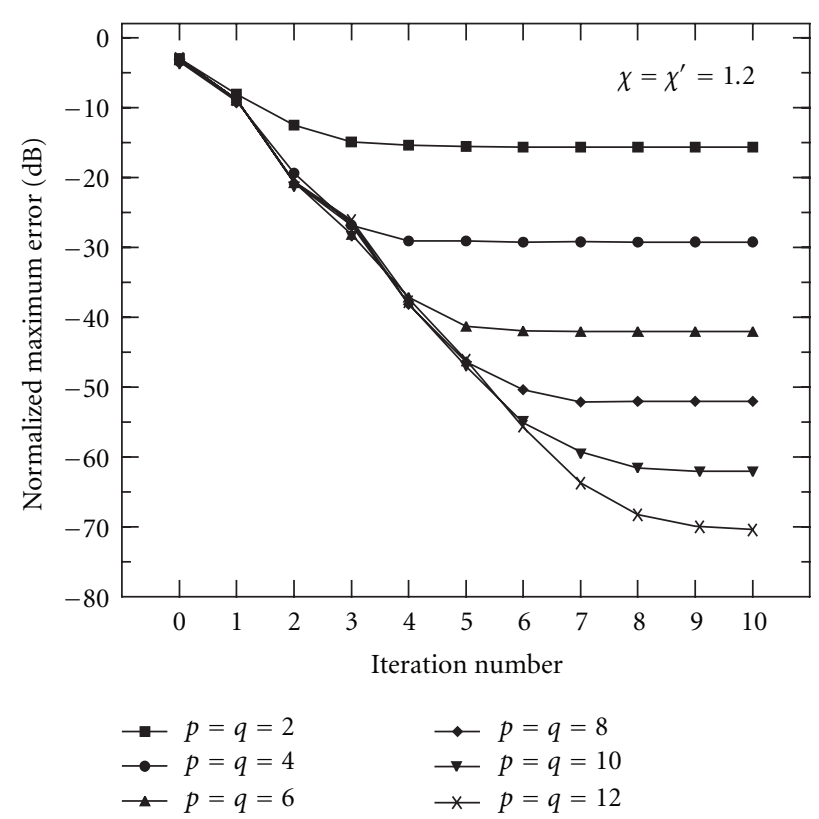

Figure 4: Normalized maximum error in the reconstruction of the uniform helicoidal samples.

enough to get a very good reconstruction. The evaluation of the maximum and mean-square errors (normalized to the maximum value of the output voltage $V$ on the cylinder) in the reconstruction of the uniform samples assesses more quantitatively the effectiveness of the proposed algorithm. They have been obtained by comparing the reconstructed uniform samples and the exact ones. As can be seen (Figures 4 and 5), on increasing the number of iterations, the errors decrease quickly until a constant saturation value is reached. Such a value decreases on increasing the retained samples number. Even better results are to be expected when the distances between the nonuniform samples and 


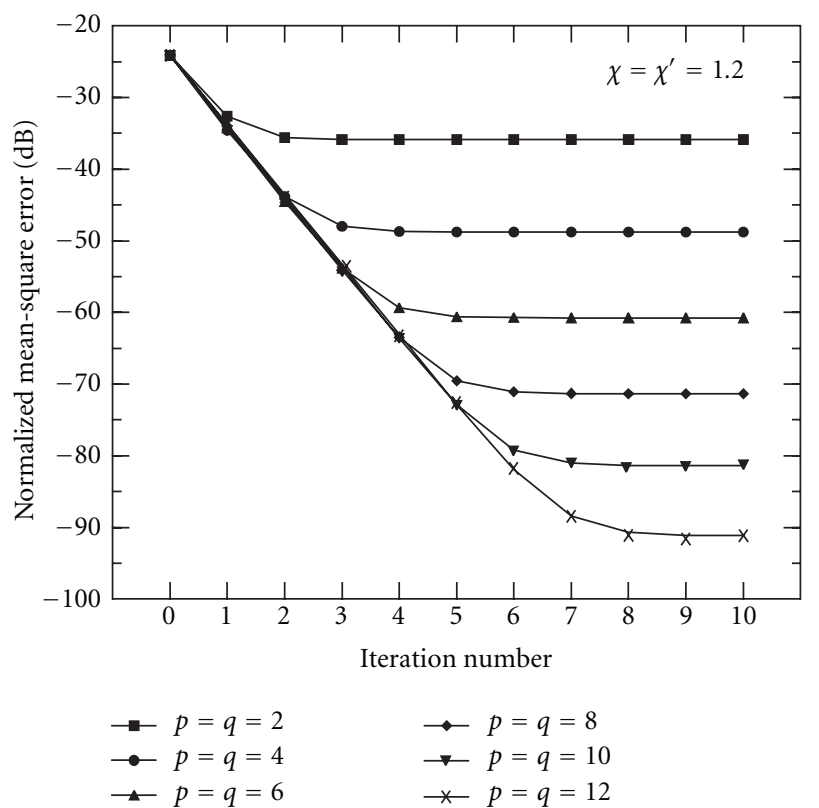

FIgURE 5: Normalized mean-square error in the reconstruction of the uniform helicoidal samples.

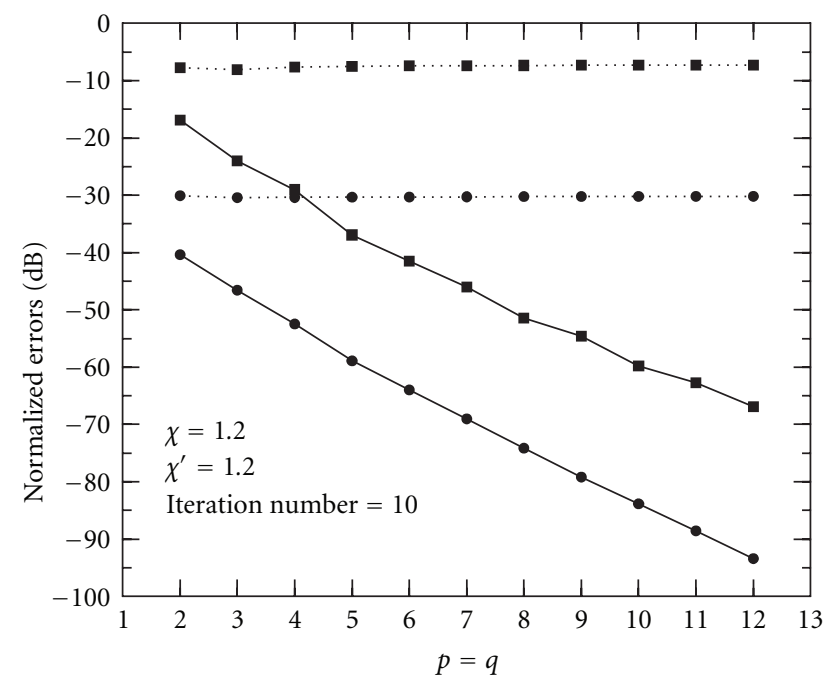

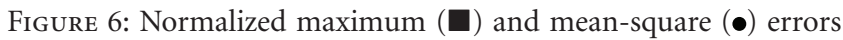
in the reconstruction of the NF data needed for the NF-FF transformation. Dotted lines: when using the nonuniform samples. Solid lines: when using the recovered helicoidal uniform samples.

the uniform ones are smaller. Moreover, Figure 6 shows the normalized maximum and mean-square errors in the reconstruction of the NF data needed to carry out the NF-FF transformation [34] both when using the directly collected nonuniform samples and the recovered uniform helicoidal ones. As can be seen, in this last case an increased accuracy of about $60 \mathrm{~dB}$ can be obtained.

The robustness of the algorithm has been assessed (see Figure 7) by corrupting the exact samples with random

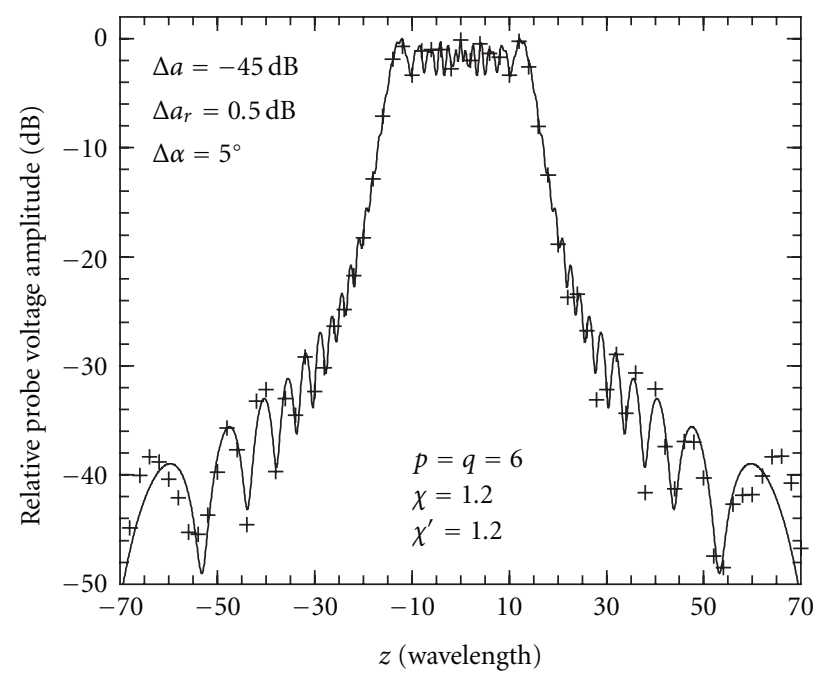

Figure 7: Amplitude of the probe voltage $V$ on the generatrix at $\varphi=90^{\circ}$. Solid line: exact. Crosses: recovered at the iteration 8 from error affected irregularly spaced NF data.

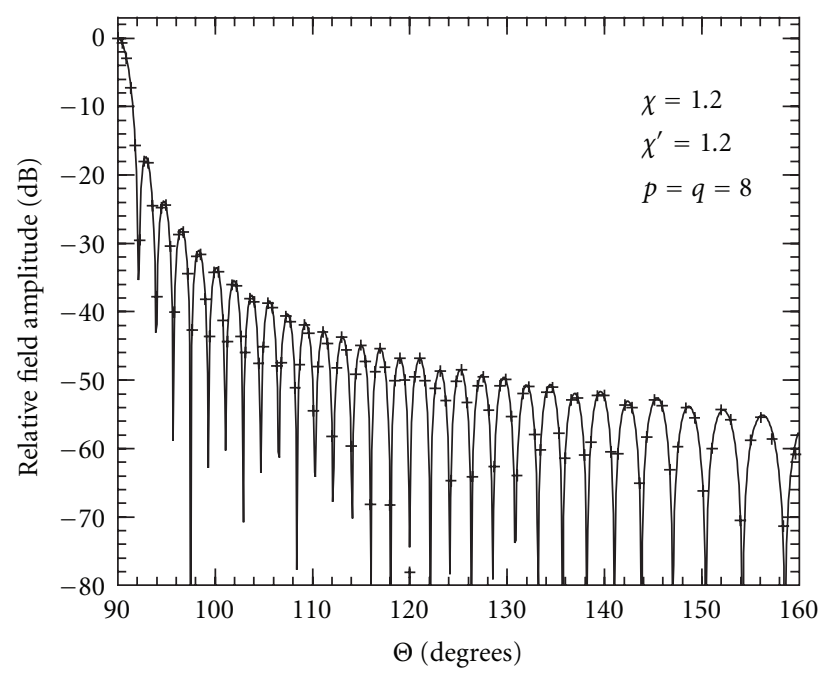

FIGURE 8: E-plane pattern. Solid line: exact. Crosses: recovered from the irregularly spaced helicoidal NF data.

errors. Both a background noise (bounded to $\Delta a$ in amplitude and with arbitrary phase) and uncertainties on the data of $\pm \Delta a_{r}$ in amplitude and $\pm \Delta \alpha$ in phase have been simulated.

At last, Figures 8 and 9 show the antenna FF pattern in the principal planes $\mathrm{E}$ and $\mathrm{H}$ reconstructed from the irregularly distributed helicoidal samples. As can be seen, the exact and recovered patterns are practically indistinguishable, thus providing an overall assessment of the proposed iterative technique.

It is worth noting that the number of employed samples (guard samples included) for reconstructing the NF data on the considered cylinder is 26817 , significantly less than that (71 680) required by the standard cylindrical scanning and by the helicoidal scanning technique [35]. 


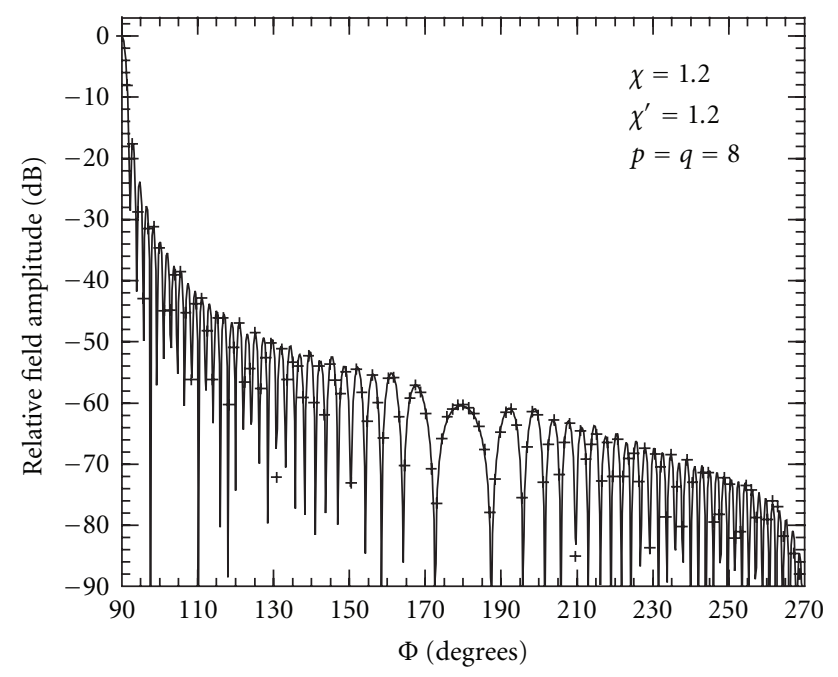

Figure 9: H-plane pattern. Solid line: exact. Crosses: recovered from the irregularly spaced helicoidal NF data.

\section{References}

[1] A. D. Yaghjian, "Overview of near-field antenna measurements," IEEE Transactions on Antennas and Propagation, vol. 34, no. 1, pp. 30-45, 1986.

[2] "Special Issue on near-field scanning techniques," IEEE Transactions on Antennas and Propagation, vol. 36, no. 6, pp. 727 901, 1988.

[3] C. Gennarelli, G. Riccio, F. D’Agostino, and F. Ferrara, NearField-Far-Field Transformation Techniques, vol. 1, CUES, Salerno, Italy, 2004.

[4] O. M. Bucci, F. D’Agostino, C. Gennarelli, G. Riccio, and C. Savarese, "NF-FF transformation with plane-polar scanning: ellipsoidal modelling of the antenna," Automatika, vol. 41, no. 3-4, pp. 159-164, 2000.

[5] F. D’Agostino, C. Gennarelli, G. Riccio, and C. Savarese, "Data reduction in the NF-FF transformation with bi-polar scanning," Microwave and Optical Technology Letters, vol. 36, no. 1, pp. 32-36, 2003.

[6] O. M. Bucci, C. Gennarelli, G. Riccio, V. Speranza, and C. Savarese, "Nonredundant representation of the electromagnetic fields over a cylinder with application to the near-fieldfar-field transformation," Electromagnetics, vol. 16, no. 3, pp. 273-290, 1996.

[7] F. D’Agostino, F. Ferrara, C. Gennarelli, G. Riccio, and C. Savarese, "NF-FF transformation with cylindrical scanning from a minimum number of data," Microwave and Optical Technology Letters, vol. 35, no. 4, pp. 264-268, 2002.

[8] O. M. Bucci, F. D’Agostino, C. Gennarelli, G. Riccio, and C. Savarese, "Data reduction in the NF-FF transformation technique with spherical scanning," Journal of Electromagnetic Waves and Applications, vol. 15, no. 6, pp. 755-775, 2001.

[9] O. M. Bucci, C. Gennarelli, and C. Savarese, "Representation of electromagnetic fields over arbitrary surfaces by a finite and nonredundant number of samples," IEEE Transactions on Antennas and Propagation, vol. 46, no. 3, pp. 351-359, 1998.

[10] J. Bolomey, B. J. Cown, G. Fine et al., "Rapid near-field antenna testing via arrays of modulated scattering probes," IEEE Transactions on Antennas and Propagation, vol. 36, no. 6, pp. 804-814, 1988.
[11] R. G. Yaccarino, L. I. Williams, and Y. Rahmat-Samii, "Linear spiral sampling for the bipolar planar near-field antenna measurement technique," IEEE Transactions on Antennas and Propagation, vol. 44, no. 7, pp. 1049-1051, 1996.

[12] O. M. Bucci, C. Gennarelli, G. Riccio, and C. Savarese, "Nonredundant NF-FF transformation with helicoidal scanning," Journal of Electromagnetic Waves and Applications, vol. 15, no. 11, pp. 1507-1519, 2001.

[13] O. M. Bucci, F. D’Agostino, C. Gennarelli, G. Riccio, and C. Savarese, "Probe compensated far-field reconstruction by near-field planar spiral scanning," IEE Proceedings: Microwaves, Antennas and Propagation, vol. 149, no. 2, pp. 119-123, 2002.

[14] O. M. Bucci, F. D’Agostino, C. Gennarelli, G. Riccio, and C. Savarese, "Near-field-far-field transformation with spherical spiral scanning," IEEE Antennas and Wireless Propagation Letters, vol. 2, pp. 263-266, 2003.

[15] F. D’Agostino, C. Gennarelli, G. Riccio, and C. Savarese, “Theoretical foundations of near-field-far-field transformations with spiral scannings," Progress in Electromagnetics Research, vol. 61, pp. 193-214, 2006.

[16] F. D’Agostino, F. Ferrara, C. Gennarelli, R. Guerriero, and M. Migliozzi, "Near-field-far-field transformation technique with helicoidal scanning for elongated antennas," Progress in Electromagnetics Research B, vol. 4, pp. 249-261, 2008.

[17] F. D’Agostino, F. Ferrara, C. Gennarelli, R. Guerriero, and M. Migliozzi, "An effective NF-FF transformation technique with planar spiral scanning tailored for quasi-planar antennas," IEEE Transactions on Antennas and Propagation, vol. 56, no. 9, pp. 2981-2987, 2008.

[18] F. D'Agostino, F. Ferrara, C. Gennarelli, R. Guerriero, M. Migliozzi, and G. Riccio, "A nonredundant near-field to far-field transformation with spherical spiral scanning for nonspherical antennas," The Open Electrical \& Electronic Engineering Journal, vol. 3, pp. 1-8, 2009.

[19] F. D’Agostino, F. Ferrara, C. Gennarelli, R. Guerriero, and M. Migliozzi, "The unified theory of near-field-far-field transformations with spiral scannings for nonspherical antennas," Progress In Electromagnetics Research B, no. 14, pp. 449-477, 2009.

[20] A. Dutt and V. Rohklin, "Fast Fourier transforms for nonequispaced data," SIAM Journal Scientific Computation, vol. 14, no. 6, pp. 1369-1393, 1993.

[21] G. Beylkin, "On the fast Fourier transform of functions with singularities," Applied and Computational Harmonic Analysis, vol. 2, no. 4, pp. 363-381, 1995.

[22] R. C. Wittmann, B. K. Alpert, and M. H. Francis, "Nearfield antenna measurements using nonideal measurement locations," IEEE Transactions on Antennas and Propagation, vol. 46, no. 5, pp. 716-722, 1998.

[23] R. C. Wittmann, B. K. Alpert, and M. H. Francis, "Nearfield, spherical-scanning antenna measurements with nonideal probe locations," IEEE Transactions on Antennas and Propagation, vol. 52, no. 8, pp. 2184-2186, 2004.

[24] F. J. Beutler, "Error-free recovery of signals from irregularly spaced samples," SIAM Review, vol. 8, no. 3, pp. 328-335, 1966.

[25] H. J. Landau, "Sampling, data transmission, and the Nyquist rate," Proceedings of the IEEE, vol. 55, no. 10, pp. 1701-1706, 1967.

[26] K. Yao and J. B. Thomas, "On some stability and interpolatory properties of non-uniform sampling expansions," IEEE Transactions on Circuit Theory, vol. 14, no. 4, pp. 404-408, 1967. 
[27] J. L. Yen, "On nonuniform sampling of bandwidth-limited signals," IRE Transactions on Circuit Theory, vol. 3, no. 4, pp. 251-257, 1956.

[28] H. J. Landau, "Necessary density conditions for sampling and interpolation of certain entire functions," Acta Mathematica, vol. 117, no. 1, pp. 37-52, 1967.

[29] O. M. Bucci, C. Gennarelli, and C. Savarese, "Interpolation of electromagnetic radiated fields over a plane by nonuniform samples," IEEE Transactions on Antennas and Propagation, vol. 41, no. 11, pp. 1501-1508, 1993.

[30] O. M. Bucci, C. Gennarelli, G. Riccio, and C. Savarese, "Electromagnetic fields interpolation from nonuniform samples over spherical and cylindrical surfaces," IEE Proceedings: Microwaves, Antennas and Propagation, vol. 141, no. 2, pp. 7784, 1994.

[31] F. Ferrara, C. Gennarelli, G. Riccio, and C. Savarese, "NF-FF transformation with cylindrical scanning from nonuniformly distributed data," Microwave and Optical Technology Letters, vol. 39, no. 1, pp. 4-8, 2003.

[32] G. H. Golub and C. F. Van Loan, Matrix Computations, J. Hopkins University Press, Baltimore, Md, USA, 1996.

[33] O. M. Bucci, G. D’Elia, and M. D. Migliore, “Advanced field interpolation from plane-polar samples: experimental verification," IEEE Transactions on Antennas and Propagation, vol. 46, no. 2, pp. 204-210, 1998.

[34] W. M. Leach Jr. and D. T. Paris, "Probe compensated near-field measurements on a cylinder," IEEE Transactions on Antennas and Propagation, vol. AP-21, no. 4, pp. 435-445, 1973.

[35] S. Costanzo and G. Di Massa, "Far-field reconstruction from phaseless near-field data on a cylindrical helix," Journal of Electromagnetic Waves and Applications, vol. 18, no. 8, pp. 1057-1071, 2004. 

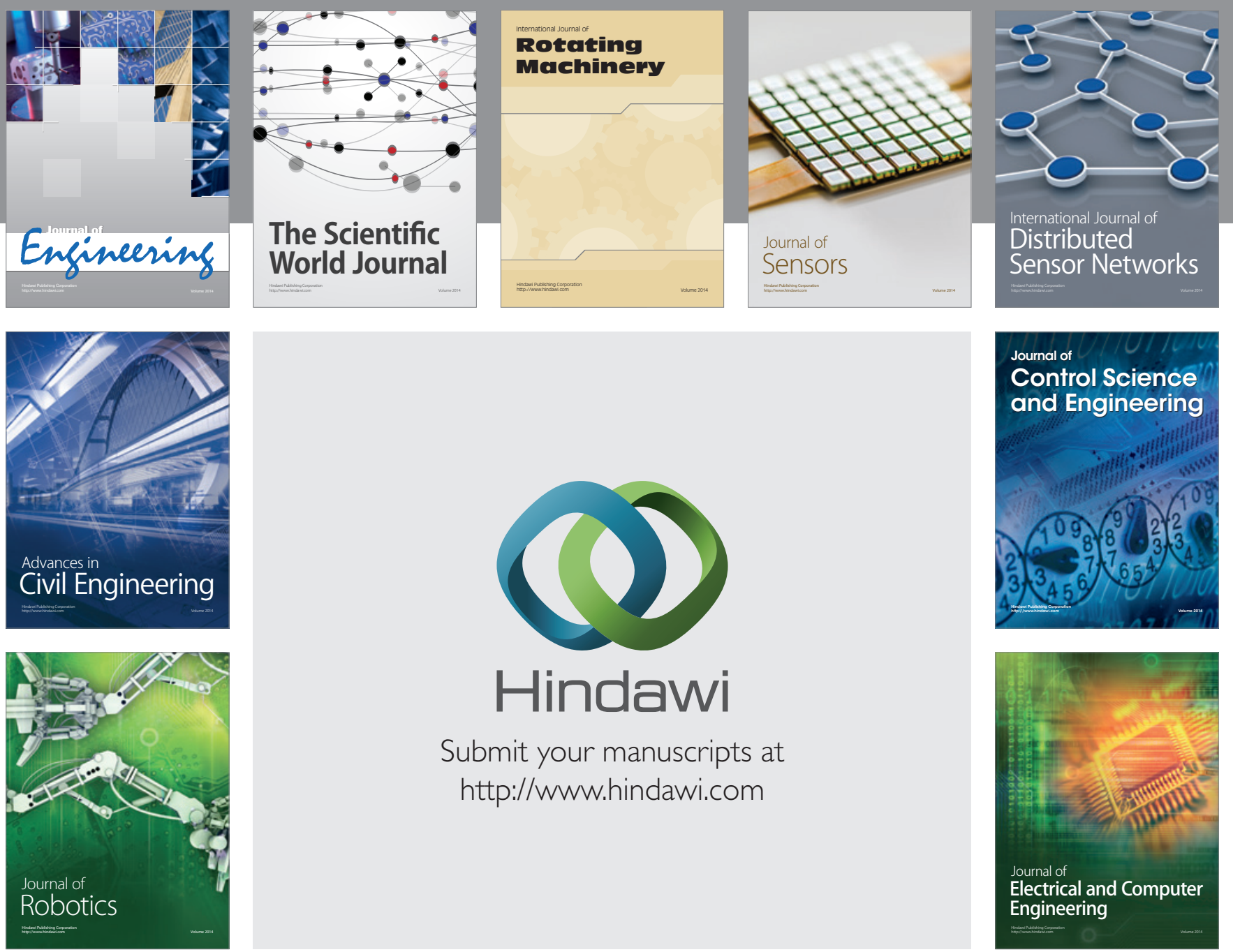

Submit your manuscripts at

http://www.hindawi.com
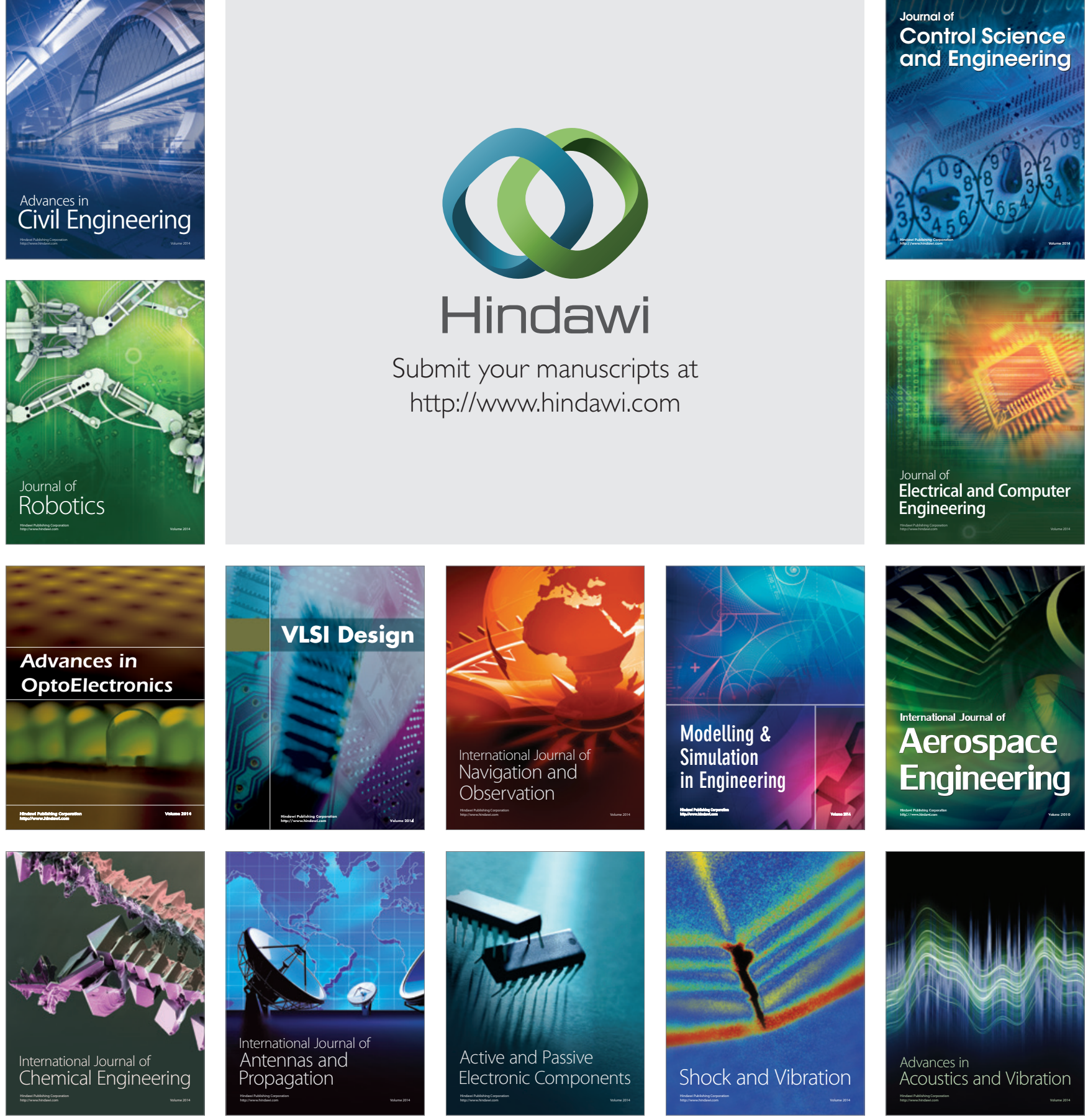